\title{
Screening Tomato Genotypes for Salt Tolerance
}

\author{
P. Arun Kumar ${ }^{1 *}$, N.N. Reddy ${ }^{2}$ and N. Jyothi Lakshmi ${ }^{2}$ \\ ${ }^{1}$ Division of Crop Improvement, ICAR-CTCRI, Thiruvananthapuram, Kerala, India \\ ${ }^{2}$ Division of Crop Science, ICAR-CRIDA, Hyderabad, Telangana, India \\ *Corresponding author
}

\begin{tabular}{|c|c|}
\hline & A B S T R A C T \\
\hline & Thirty three genotypes of tomato including three checks (NS-515, Arka Rakshak and US- \\
\hline & $\begin{array}{l}\text { 440) were analysed to determine their tolerance levels against salt stress. Four different } \\
\text { concentrations of } \mathrm{NaCl} \text { viz., } 50 \mathrm{mM}, 75 \mathrm{mM}, 100 \mathrm{mM} \text { and } 150 \mathrm{mM} \text { were used along with }\end{array}$ \\
\hline $\begin{array}{l}\text { Tomato, NaCl, } \\
\text { Screening. }\end{array}$ & $\begin{array}{l}\text { control. Significant varietal and or salinity treatment effects were recorded on germination } \\
\text { percentage, germination rate, root length, shoot length, root dry weight and shoot dry }\end{array}$ \\
\hline Article Info & $\begin{array}{l}\text { weight. With increasing concentration of } \mathrm{NaCl} \text { growth parameters of tomato genotypes } \\
\text { decreased proportionately. Among the commercial checks NS-516 while among crosses }\end{array}$ \\
\hline $\begin{array}{l}\text { Accepted: } \\
\text { 10 September } 2017 \\
\text { Available Online: } \\
10 \text { November } 2017\end{array}$ & $\begin{array}{l}\text { EC- } 619982 \times \text { EC- } 520078 \text {, EC- } 619982 \times \text { Pusa Ruby, EC- } 620428 \times \text { Punjab Chhuhara, EC- } \\
620360 \times \text { EC- } 620557 \text {, EC- } 620407 \times \text { Punjab Chhuhara and EC- } 608415 \times \text { Arka Saurabh } \\
\text { responded positively against different } \mathrm{NaCl} \text { concentrations. Confirmatory studies may be } \\
\text { performed to access their potential under pot cultures before the internal physiological } \\
\text { investigations for their variable response. }\end{array}$ \\
\hline
\end{tabular}

\section{Introduction}

Salinity is one of the most severe abiotic factors, limiting agricultural production in arid and semi-arid regions. Excessive salinity reduces the productivity of many agricultural crops. Salt stress affects major processes like germination, speed of germination, root/shoot dry weight and $\mathrm{Na}+/ \mathrm{K}+$ ratio in root and shoot (Parida and Das, 2005). Excess salt in soil solution adversely affect plant growth, either through osmotic inhibition of root water uptake or by specific ion effect. Seed germination and early seedling growth are the most sensitive stages to environmental stresses such as salinity (Sivritepe et al., 2003). Salinity may cause significant reductions in the rate and final percentage of germination, which in turn may lead to uneven stand establishment and reduced crop yields (Foolad et al., 1999). The problem of soil salinity is now increasing at a rate of $10 \%$ annually (Flowers, 2004; Foolad, 2007). It is expected that by 2050 , more than $50 \%$ of the land available for agriculture will be lost because of salinity (Hasanuzzaman et al., 2014).

Tomato is one of the widely grown vegetables in the world. It is consumed in fresh form as salad and in various processed forms like soup, sauce, ketchup, paste, puree, powder and canned whole fruit etc. and tops the list of processed vegetables. Tomato is moderately sensitive to salinity (Peralta et al., 2005), although considerable differences between 
cultivars may be observed (Allen et al., 1998). It can tolerate salinity up to 2.5-2.9 dS $\mathrm{m}-1$ in the root zone without yield losses (Sonneveld and Vander Burg, 1991). The exact salinity level may vary depending on cultivar sensitivity (Caro et al., 1991) and environmental conditions (Karlberg et al., 2006). Therefore, the experiment was undertaken to evaluate the ability of tomato for growing under different saline conditions and screen them for salinity tolerance.

\section{Materials and Methods}

The study was conducted at Horticulture Laboratory of Central Research Institute for Dry Land Agriculture (CRIDA), Hyderabad during 2016. The method suggested by Panchalingam (1983) and Babu et al., (1985) was followed to screen the genotypes against salt stress environment under laboratory conditions. Thirty crosses of tomato along with three commercial checks viz., Arka Rakshak, US-440 and NS-516 were screened by employing germination test in an osmotic solution of $\mathrm{NaCl}$ (sodium chloride). Simultaneously, all the accessions were allowed to germinate in distilled water. Three replications of 10 seeds for each accession were counted and distributed over two layers of paper towel $(11 \times 11 \mathrm{~cm})$ previously moistened with water equivalent to three times the dry weight of the paper and tied both ends with rubber band and kept in a plastic tray with different concentrations (50mM, 75mM, $100 \mathrm{M}$ and $150 \mathrm{mM}$ ) of sodium chloride $(\mathrm{NaCl})$. Germination percentage was recorded for every 7 days. At the end of the $21^{\text {st }}$ day, final germination per cent, germination rate, root length, shoot length, root dry weight and shoot weight were recorded in $\mathrm{NaCl}$ solution as against the distilled water was calculated. The experiment was designed as a completely randomized design with two factors. The first factor was the genotypes and the second one is external salt stress. The sheets were rolled and placed vertically in plastic beaker, covered with polythene bag and placed at $25 \pm 1^{\circ} \mathrm{C}$ in an illuminated germinator. A seed was considered to be germinated when the emerging radicle elongated to $1 \mathrm{~mm}$. Radicle length, hypocotyl length, seedling fresh weight and dry weight were calculated as described by Uniyal et al., (1998). Observations were recorded on germination percentage, germination rate, root length $(\mathrm{cm})$, shoot length $(\mathrm{cm})$, root dry weight $(\mathrm{mg})$ and shoot dry weight (mg). Analysis of variance was carried out as described by Steel et al., (1997). Statistical significance of means was tested by SPSS package.

\section{Results and Discussion}

The analysis of variance showed significant differences among the genotypes and treatments (Table 1). Significant differences were observed under different $\mathrm{NaCl}$ concentrations of $50 \mathrm{mM}, 75 \mathrm{mM}, 100 \mathrm{M}$ and $150 \mathrm{mM}$.

\section{Germination percentage $(\%)$}

A decline in seed germination percentage with increasing stress has been recorded (Table 2). Significant differences were observed for germination percentage between the genotypes and different $\mathrm{NaCl}$ concentrations. Irrespective of $\mathrm{NaCl}$ concentrations, NS-516 recorded significantly maximum germination percentage $(86.08 \%)$ compared to other genotypes. Germination percentage was lowest $(34.31 \%)$ in the cross EC-620428 $\times$ EC-620557. At $50 \mathrm{mM}$ concentration of $\mathrm{NaCl}$, the cross NS-516 exhibited maximum (87.93\%) and EC-620428 $\times$ EC-520078 exhibited minimum $(32.82 \%)$ values. While at $75 \mathrm{mM}$ concentration genotypes NS-516 (85.66\%) and EC-620428 $\times$ EC-520078 (26.24\%) exhibited highest and lowest values respectively. In case of $100 \mathrm{mM}$ 
concentration NS-516 (85.54\%), EC-620428 $\times$ EC-520078 (26.24\%) responded as maximum and minimum germination percentage respectively. With $150 \mathrm{mM}$ concentration of $\mathrm{NaCl}, \mathrm{NS}-516$ (82.87\%) responded maximum and minimum in EC$620428 \times$ EC-620557 (19.82\%).

It was observed that germination percentage with decreasing water potential of the environment probably was triggered by the low hydraulic conductivity where, $\mathrm{NaCl}$ makes water unavailable to seeds, affecting the imbibition process of the seed which is fundamental for germination.

Results of the current study were in agreement with findings of El-Saifi et al., (2010), Souguir et al., (2013), Ravi et al., (2011) and Alejandra et al., (2010).

\section{Germination rate}

Significant differences were observed for germination rate between the genotypes and $\mathrm{NaCl}$ concentrations (Table 3). Irrespective of salt concentrations, NS-516 recorded maximum germination rate (8.27) compared to other genotypes. The germination rate was lowest (3.04\%) in EC-620428 × EC-520078. At $50 \mathrm{mM}$ concentration of $\mathrm{NaCl}$, maximum and minimum germination rates were recorded in NS-516 (8.91) and EC-620428 $\times$ EC-520078 (2.48\%) respectively.
While at $75 \mathrm{mM}$ concentration, genotype NS516 (8.26) and EC-620428 × EC-520078 (2.44) exhibited maximum and minimum values respectively. In case of $100 \mathrm{mM}$ concentration, maximum and minimum germination rate recorded in NS-516 (7.65) and EC-620428 × EC-520078 (2.33) while with $150 \mathrm{mM}$ maximum and minimum germination rates were recorded in NS-516 (7.43\%) and EC-620407 × Punjab Chhuhara (1.74) respectively. The rate of germination seems to be more sensitive to water stress than final germination percentage caused a significant increase in mean germination time and decrease in germination. Present studies are intensely supported by the finding of ElSaifi et al., (2010), Souguir et al., (2013), Ravi et al., (2011) and Alejandra et al., (2010).

\section{Root length (cm)}

Root length is an important trait against salt stress in plant varieties, with longer root growth has resistant ability for salt. Early and rapid elongation of roots is an important indication of tolerance. Ability of continued elongation of root under water stress and longer root length at deeper layer are useful in extracting water in upland conditions (Kim et al., 2001, Narayan, 1991). The plant embryo grows at germination and progresses radicles that become the primary roots and penetrate down into the soil.

Table.1 Analysis of variance for six characters in tomato

\begin{tabular}{|c|c|c|c|c|c|c|c|}
\hline \multicolumn{7}{|c|}{ Mean sum of squares } \\
\hline $\begin{array}{c}\text { Source of } \\
\text { variation }\end{array}$ & df & $\begin{array}{c}\text { Germination } \\
\mathbf{\%}\end{array}$ & $\begin{array}{c}\text { Germinatio } \\
\text { n rate }\end{array}$ & $\begin{array}{c}\text { Root } \\
\text { length }\end{array}$ & $\begin{array}{c}\text { Shoot } \\
\text { length }\end{array}$ & $\begin{array}{c}\text { Shoot dry } \\
\text { weight }\end{array}$ & $\begin{array}{c}\text { Root dry } \\
\text { weight }\end{array}$ \\
\hline Treatment (A) & 4 & $1928.15^{* *}$ & $15.68^{* *}$ & $15.53^{* *}$ & 13.19 & $44.65^{* *}$ & $904.15^{* *}$ \\
\hline Genotype (B) & 32 & $325.51^{* *}$ & $0.83^{* *}$ & $1.23^{* *}$ & $12.55^{* *}$ & $6.09^{* *}$ & $895.30^{* *}$ \\
\hline $\mathrm{A} \times \mathrm{B}$ & 156 & 561.61 & 6.28 & 5.88 & 17.49 & 12.63 & 1489.77 \\
\hline Error & 424 & 26.75 & 0.14 & 0.11 & 0.10 & 0.10 & 6.91 \\
\hline
\end{tabular}


Table.2 Effect of different concentration of $\mathrm{NaCl}$ (control, 50mM, 75mM, 100mM and 150mM on germination percentage of tomato genotypes

\begin{tabular}{|c|c|c|c|c|c|c|}
\hline \multirow{3}{*}{ Crosses/Hybrids } & \multicolumn{6}{|c|}{ Germination percent $(\%)$} \\
\hline & \multicolumn{6}{|c|}{ Treatments } \\
\hline & Control & $50 \mathrm{mM}$ & $\begin{array}{c}75 \mathrm{~m} \\
\mathrm{M}\end{array}$ & $100 \mathrm{mM}$ & $150 \mathrm{mM}$ & Mean \\
\hline EC-620407 × Arka Saurabh & 57.08 & 52.45 & 54.34 & 42.10 & 38.26 & 48.85 \\
\hline EC-620407 × Arka Abha & 65.38 & 64.47 & 55.88 & 53.70 & 49.90 & 57.87 \\
\hline EC-620407 × Punjab Chhuhara & 64.90 & 54.08 & 47.51 & 41.49 & 41.44 & 49.88 \\
\hline EC-620407 × Pusa Ruby & 40.71 & 36.13 & 35.02 & 33.34 & 33.31 & 35.70 \\
\hline EC-620407 × EC-520078 & 71.04 & 65.48 & 61.47 & 53.24 & 52.92 & 60.83 \\
\hline EC-620407 × EC-620557 & 76.33 & 67.04 & 66.72 & 56.17 & 54.99 & 64.25 \\
\hline EC-620428 × Arka Saurabh & 46.09 & 45.34 & 40.89 & 35.56 & 32.57 & 40.09 \\
\hline EC-620428 × Arka Abha & 44.67 & 35.59 & 34.76 & 33.90 & 30.29 & 35.84 \\
\hline EC-620428 × Punjab Chhuhara & 58.02 & 56.31 & 44.98 & 43.05 & 42.67 & 49.01 \\
\hline EC-620428 × Pusa Ruby & 76.01 & 69.69 & 66.57 & 63.83 & 60.76 & 67.37 \\
\hline EC-620428 $\times$ EC-520078 & 67.78 & 32.82 & 28.09 & 26.24 & 24.82 & 35.95 \\
\hline EC-620428 × EC-620557 & 43.22 & 41.75 & 38.49 & 28.25 & 19.82 & 34.31 \\
\hline EC-620360 × Arka Saurabh & 64.55 & 62.69 & 60.01 & 48.80 & 46.17 & 56.44 \\
\hline EC-620360 × Arka Abha & 82.66 & 67.23 & 62.03 & 44.25 & 43.81 & 59.99 \\
\hline EC-620360 × Punjab Chhuhara & 71.64 & 46.74 & 45.67 & 32.39 & 33.15 & 45.92 \\
\hline EC-620360 × Pusa Ruby & 61.30 & 46.52 & 42.53 & 39.90 & 34.09 & 44.87 \\
\hline EC-620360 × EC-520078 & 52.38 & 47.86 & 41.21 & 40.25 & 42.01 & 44.74 \\
\hline EC-620360 × EC-620557 & 52.95 & 51.93 & 51.34 & 43.08 & 39.02 & 47.66 \\
\hline EC-608415 × Arka Saurabh & 61.01 & 55.07 & 53.57 & 49.69 & 40.39 & 51.95 \\
\hline EC-608415 × Arka Abha & 66.84 & 61.97 & 57.44 & 55.47 & 53.67 & 59.08 \\
\hline EC-608415 × Punjab Chhuhara & 69.30 & 63.65 & 61.49 & 53.31 & 48.35 & 59.22 \\
\hline EC-608415 × Pusa Ruby & 83.56 & 54.21 & 53.80 & 38.94 & 33.24 & 52.75 \\
\hline EC-608415 × EC-520078 & 67.30 & 46.61 & 38.76 & 32.85 & 21.28 & 41.36 \\
\hline EC-608415 × EC-620557 & 63.01 & 53.59 & 50.77 & 35.91 & 27.90 & 46.24 \\
\hline EC-619982 × Arka Saurabh & 57.46 & 50.67 & 46.69 & 36.59 & 30.96 & 44.47 \\
\hline EC-619982 × Arka Abha & 79.90 & 63.64 & 61.73 & 56.62 & 40.88 & 60.55 \\
\hline EC-619982 × Punjab Chhuhara & 85.15 & 64.67 & 60.38 & 44.59 & 43.78 & 59.71 \\
\hline EC-619982 × Pusa Ruby & 82.66 & 72.48 & 66.88 & 64.59 & 52.54 & 67.83 \\
\hline EC-619982 × EC-520078 & 84.93 & 73.94 & 72.76 & 61.68 & 54.72 & 69.61 \\
\hline EC-619982 × EC-620557 & 74.62 & 70.67 & 64.19 & 59.80 & 44.23 & 62.70 \\
\hline US-440 & 89.81 & 85.01 & 84.47 & 82.84 & 70.36 & 82.50 \\
\hline Arka Rakshak & 89.96 & 85.81 & 82.75 & 80.52 & 77.67 & 83.34 \\
\hline NS-516 & 88.42 & 87.93 & 85.66 & 85.54 & 82.87 & 86.08 \\
\hline \multirow[t]{2}{*}{ Mean } & 67.89 & 58.60 & 55.11 & 48.43 & 43.72 & \\
\hline & \multicolumn{3}{|c|}{ S.Em \pm} & \multicolumn{3}{|c|}{$\mathrm{CD}(0.01)$} \\
\hline Treatment (A) & \multicolumn{3}{|c|}{1.33} & \multicolumn{3}{|c|}{3.72} \\
\hline Genotype (B) & \multicolumn{3}{|c|}{0.52} & \multicolumn{3}{|c|}{1.45} \\
\hline$A \times B$ & \multicolumn{3}{|c|}{2.99} & \multicolumn{3}{|c|}{8.31} \\
\hline
\end{tabular}


Table.3 Effect of different concentration of $\mathrm{NaCl}$ (control, 50mM, 75mM, $100 \mathrm{M}$ and 150mM on germination rate of tomato genotypes

\begin{tabular}{|c|c|c|c|c|c|c|}
\hline \multirow{3}{*}{ Crosses/Hybrids } & \multicolumn{6}{|c|}{ Germination rate $(\%)$} \\
\hline & \multicolumn{6}{|c|}{ Treatments } \\
\hline & Control & $50 \mathrm{mM}$ & $\begin{array}{c}75 \mathrm{~m} \\
\mathrm{M}\end{array}$ & $100 \mathrm{mM}$ & $150 \mathrm{mM}$ & Mean \\
\hline EC-620407 × Arka Saurabh & 4.80 & 4.66 & 4.40 & 3.96 & 3.67 & 4.30 \\
\hline EC-620407 × Arka Abha & 5.73 & 5.71 & 4.93 & 4.80 & 4.57 & 5.15 \\
\hline EC-620407 × Punjab Chhuhara & 5.05 & 4.79 & 4.48 & 3.99 & 1.74 & 4.01 \\
\hline EC-620407 × Pusa Ruby & 3.85 & 3.33 & 3.30 & 3.05 & 3.04 & 3.31 \\
\hline EC-620407 × EC-520078 & 7.02 & 6.59 & 5.65 & 4.71 & 2.83 & 5.36 \\
\hline EC-620407 × EC-620557 & 6.48 & 6.06 & 5.89 & 5.57 & 4.74 & 5.75 \\
\hline EC-620428 × Arka Saurabh & 3.59 & 4.82 & 3.55 & 3.52 & 3.37 & 3.77 \\
\hline EC-620428 × Arka Abha & 3.40 & 3.03 & 2.91 & 2.89 & 2.66 & 2.98 \\
\hline EC-620428 × Punjab Chhuhara & 4.46 & 4.56 & 4.51 & 4.07 & 3.09 & 4.14 \\
\hline EC-620428 × Pusa Ruby & 6.51 & 6.34 & 6.24 & 5.84 & 3.64 & 5.71 \\
\hline EC-620428 × EC-520078 & 5.76 & 2.48 & 2.44 & 2.33 & 2.18 & 3.04 \\
\hline EC-620428 × EC-620557 & 4.55 & 4.16 & 4.00 & 2.35 & 1.69 & 3.35 \\
\hline EC-620360 × Arka Saurabh & 6.25 & 5.86 & 5.69 & 4.22 & 3.73 & 5.15 \\
\hline EC-620360 × Arka Abha & 6.44 & 5.79 & 5.23 & 5.00 & 4.00 & 5.29 \\
\hline EC-620360 × Punjab Chhuhara & 4.47 & 4.39 & 4.14 & 3.72 & 2.96 & 3.94 \\
\hline EC-620360 × Pusa Ruby & 4.71 & 4.47 & 4.44 & 3.70 & 2.51 & 3.97 \\
\hline EC-620360 × EC-520078 & 4.42 & 4.37 & 4.23 & 3.78 & 3.02 & 3.96 \\
\hline EC-620360 × EC-620557 & 4.75 & 4.55 & 4.48 & 3.97 & 3.89 & 4.33 \\
\hline EC-608415 × Arka Saurabh & 6.03 & 5.78 & 3.75 & 3.59 & 5.28 & 4.89 \\
\hline EC-608415 × Arka Abha & 6.57 & 6.39 & 6.21 & 5.74 & 5.18 & 6.02 \\
\hline EC-608415 × Punjab Chhuhara & 5.97 & 5.43 & 5.60 & 5.28 & 4.86 & 5.43 \\
\hline EC-608415 × Pusa Ruby & 5.11 & 4.88 & 4.33 & 4.27 & 3.17 & 4.35 \\
\hline EC-608415 × EC-520078 & 4.48 & 3.98 & 3.51 & 3.48 & 2.99 & 3.69 \\
\hline EC-608415 × EC-620557 & 7.85 & 3.53 & 3.32 & 2.36 & 2.62 & 3.94 \\
\hline EC-619982 × Arka Saurabh & 7.96 & 6.48 & 5.34 & 2.91 & 2.15 & 4.97 \\
\hline EC-619982 × Arka Abha & 6.45 & 5.88 & 5.56 & 3.98 & 3.41 & 5.06 \\
\hline EC-619982 × Punjab Chhuhara & 7.46 & 5.75 & 4.28 & 4.10 & 3.25 & 4.97 \\
\hline EC-619982 × Pusa Ruby & 7.40 & 6.88 & 6.32 & 5.22 & 5.11 & 6.19 \\
\hline EC-619982 × EC-520078 & 7.12 & 6.49 & 5.86 & 5.49 & 5.27 & 6.05 \\
\hline EC-619982 × EC-620557 & 7.18 & 7.02 & 5.60 & 5.35 & 4.81 & 5.99 \\
\hline US-440 & 8.78 & 8.52 & 8.02 & 7.59 & 7.38 & 8.06 \\
\hline Arka Rakshak & 8.68 & 8.03 & 7.52 & 7.04 & 6.99 & 7.65 \\
\hline NS-516 & 9.11 & 8.91 & 8.26 & 7.65 & 7.43 & 8.27 \\
\hline \multirow[t]{2}{*}{ Mean } & 6.01 & 5.45 & 4.97 & 4.41 & 3.86 & \\
\hline & \multicolumn{3}{|c|}{ S.Em \pm} & \multicolumn{3}{|c|}{ CD (0.01) } \\
\hline Treatment (A) & \multicolumn{3}{|c|}{0.096} & \multicolumn{3}{|c|}{0.267} \\
\hline Genotype (B) & \multicolumn{3}{|c|}{0.037} & \multicolumn{3}{|c|}{0.104} \\
\hline $\mathbf{A} \times \mathbf{B}$ & \multicolumn{3}{|c|}{0.214} & \multicolumn{3}{|c|}{0.596} \\
\hline
\end{tabular}


Table.4 Effect of different concentration of $\mathrm{NaCl}$ (control, 50mM, 75mM, $100 \mathrm{M}$ and 150mM on root length of tomato genotypes

\begin{tabular}{|c|c|c|c|c|c|c|}
\hline \multirow{3}{*}{ Crosses/Hybrids } & \multicolumn{6}{|c|}{ Root length $(\mathrm{cm})$} \\
\hline & \multicolumn{6}{|c|}{ Treatments } \\
\hline & Control & $\begin{array}{c}\text { 50m } \\
M\end{array}$ & $\begin{array}{c}75 \mathrm{~m} \\
\mathrm{M}\end{array}$ & $100 \mathrm{mM}$ & $150 \mathrm{mM}$ & Mean \\
\hline EC-620407 × Arka Saurabh & 5.69 & 5.67 & 5.27 & 4.41 & 4.39 & 5.09 \\
\hline EC-620407 × Arka Abha & 5.02 & 4.38 & 3.92 & 3.26 & 2.82 & 3.88 \\
\hline EC-620407 × Punjab Chhuhara & 5.01 & 5.03 & 4.52 & 3.91 & 3.69 & 4.43 \\
\hline EC-620407 × Pusa Ruby & 4.33 & 4.20 & 3.54 & 3.02 & 2.62 & 3.54 \\
\hline EC-620407 × EC-520078 & 6.11 & 5.56 & 5.41 & 4.89 & 4.73 & 5.34 \\
\hline EC-620407 × EC-620557 & 5.35 & 5.04 & 4.15 & 3.51 & 3.10 & 4.23 \\
\hline EC-620428 × Arka Saurabh & 4.96 & 4.48 & 4.20 & 3.58 & 3.34 & 4.11 \\
\hline EC-620428 × Arka Abha & 6.03 & 5.91 & 5.75 & 4.98 & 4.64 & 5.46 \\
\hline EC-620428 × Punjab Chhuhara & 7.87 & 7.24 & 6.84 & 6.32 & 5.97 & 6.85 \\
\hline EC-620428 × Pusa Ruby & 8.36 & 8.16 & 7.40 & 7.05 & 6.82 & 7.56 \\
\hline EC-620428 × EC-520078 & 4.25 & 3.63 & 3.40 & 2.86 & 2.74 & 3.38 \\
\hline EC-620428 × EC-620557 & 6.01 & 5.39 & 5.14 & 4.61 & 4.05 & 5.04 \\
\hline EC-620360 × Arka Saurabh & 3.51 & 2.88 & 2.16 & 2.32 & 1.50 & 2.47 \\
\hline EC-620360 × Arka Abha & 4.89 & 4.56 & 3.41 & 3.03 & 2.82 & 3.74 \\
\hline EC-620360 × Punjab Chhuhara & 3.51 & 2.92 & 2.65 & 2.35 & 2.15 & 2.72 \\
\hline EC-620360 × Pusa Ruby & 5.06 & 4.41 & 4.19 & 3.44 & 1.93 & 3.81 \\
\hline EC-620360 × EC-520078 & 3.61 & 3.31 & 3.13 & 2.41 & 2.11 & 2.91 \\
\hline EC-620360 × EC-620557 & 5.26 & 4.82 & 4.33 & 3.34 & 3.19 & 4.19 \\
\hline EC-608415 × Arka Saurabh & 4.28 & 4.25 & 3.80 & 3.51 & 2.74 & 3.72 \\
\hline EC-608415 × Arka Abha & 5.08 & 4.50 & 4.00 & 3.61 & 3.31 & 4.10 \\
\hline EC-608415 × Punjab Chhuhara & 4.88 & 4.60 & 4.21 & 3.58 & 3.38 & 4.13 \\
\hline EC-608415 × Pusa Ruby & 5.64 & 4.70 & 4.51 & 4.13 & 3.27 & 4.45 \\
\hline EC-608415 × EC-520078 & 7.11 & 6.55 & 6.39 & 5.42 & 4.57 & 6.01 \\
\hline EC-608415 × EC-620557 & 6.30 & 5.94 & 5.22 & 5.02 & 4.21 & 5.34 \\
\hline EC-619982 × Arka Saurabh & 6.95 & 6.75 & 6.47 & 5.76 & 5.58 & 6.30 \\
\hline EC-619982 × Arka Abha & 6.20 & 5.64 & 5.44 & 4.69 & 4.61 & 5.32 \\
\hline EC-619982 × Punjab Chhuhara & 5.74 & 5.39 & 5.28 & 4.64 & 4.13 & 5.04 \\
\hline EC-619982 × Pusa Ruby & 8.03 & 7.32 & 6.95 & 6.62 & 5.79 & 6.94 \\
\hline EC-619982 × EC-520078 & 2.62 & 2.09 & 1.99 & 1.39 & 1.26 & 1.87 \\
\hline EC-619982 × EC-620557 & 6.07 & 5.66 & 5.55 & 4.89 & 4.55 & 5.34 \\
\hline US-440 & 7.73 & 7.52 & 7.34 & 6.45 & 4.91 & 6.79 \\
\hline Arka Rakshak & 7.29 & 7.21 & 7.14 & 6.65 & 6.15 & 6.89 \\
\hline NS-516 & 8.08 & 7.90 & 7.58 & 6.88 & 5.98 & 7.28 \\
\hline \multirow[t]{2}{*}{ Mean } & 5.66 & 5.26 & 4.89 & 4.32 & 3.85 & \\
\hline & \multicolumn{3}{|c|}{ S.Em \pm} & \multicolumn{3}{|c|}{ CD (0.01) } \\
\hline Treatment (A) & \multicolumn{3}{|c|}{0.084} & \multicolumn{3}{|c|}{0.234} \\
\hline Genotype (B) & \multicolumn{3}{|c|}{0.033} & \multicolumn{3}{|c|}{0.091} \\
\hline $\mathbf{A} \times \mathbf{B}$ & \multicolumn{3}{|c|}{0.188} & \multicolumn{3}{|c|}{0.523} \\
\hline
\end{tabular}


Table.5 Effect of different concentration of $\mathrm{NaCl}$ (control, 50mM, 75mM, $100 \mathrm{M}$ and 150mM on shoot length $(\mathrm{cm})$ of tomato genotypes

\begin{tabular}{|c|c|c|c|c|c|c|}
\hline \multirow{3}{*}{ Crosses/Hybrids } & \multicolumn{6}{|c|}{ Shoot length $(\mathrm{cm})$} \\
\hline & \multicolumn{6}{|c|}{ Treatments } \\
\hline & Control & $50 \mathrm{mM}$ & $\begin{array}{c}75 \mathrm{~m} \\
\mathrm{M}\end{array}$ & $100 \mathrm{mM}$ & $150 \mathrm{mM}$ & Mean \\
\hline EC-620407 × Arka Saurabh & 4.85 & 4.43 & 3.76 & 3.34 & 3.17 & 3.91 \\
\hline EC-620407 × Arka Abha & 3.85 & 3.37 & 3.30 & 2.49 & 2.41 & 3.09 \\
\hline EC-620407 × Punjab Chhuhara & 4.77 & 4.54 & 4.23 & 3.72 & 3.60 & 4.17 \\
\hline EC-620407 × Pusa Ruby & 8.23 & 7.82 & 7.39 & 6.72 & 6.52 & 6.74 \\
\hline EC-620407 × EC-520078 & 5.27 & 4.73 & 4.27 & 3.57 & 3.45 & 4.85 \\
\hline EC-620407 × EC-620557 & 7.24 & 6.77 & 6.40 & 5.46 & 5.42 & 5.82 \\
\hline EC-620428 × Arka Saurabh & 5.04 & 2.50 & 1.51 & 1.39 & 1.25 & 2.78 \\
\hline EC-620428 × Arka Abha & 3.60 & 3.26 & 2.47 & 2.50 & 1.85 & 2.73 \\
\hline EC-620428 × Punjab Chhuhara & 5.11 & 4.65 & 4.31 & 3.59 & 3.42 & 3.93 \\
\hline EC-620428 × Pusa Ruby & 3.69 & 2.03 & 1.57 & 1.49 & 1.16 & 2.27 \\
\hline EC-620428 × EC-520078 & 8.54 & 8.00 & 7.79 & 5.40 & 2.01 & 6.35 \\
\hline EC-620428 × EC-620557 & 9.37 & 6.15 & 5.35 & 4.68 & 4.44 & 6.00 \\
\hline EC-620360 × Arka Saurabh & 6.64 & 6.00 & 5.93 & 5.41 & 5.09 & 5.81 \\
\hline EC-620360 × Arka Abha & 2.67 & 1.60 & 1.54 & 1.32 & 1.20 & 2.48 \\
\hline EC-620360 × Punjab Chhuhara & 6.67 & 3.38 & 3.29 & 2.63 & 2.35 & 2.65 \\
\hline EC-620360 × Pusa Ruby & 4.16 & 4.14 & 3.96 & 3.36 & 3.07 & 3.74 \\
\hline EC-620360 × EC-520078 & 4.96 & 4.17 & 3.47 & 2.91 & 2.78 & 3.66 \\
\hline EC-620360 × EC-620557 & 10.77 & 10.09 & 8.93 & 8.91 & 8.91 & 9.49 \\
\hline EC-608415 × Arka Saurabh & 7.20 & 6.58 & 6.33 & 6.02 & 5.41 & 6.31 \\
\hline EC-608415 × Arka Abha & 2.02 & 1.61 & 1.40 & 1.60 & 0.41 & 2.48 \\
\hline EC-608415 × Punjab Chhuhara & 7.37 & 5.27 & 4.57 & 4.19 & 4.05 & 4.02 \\
\hline EC-608415 × Pusa Ruby & 6.99 & 6.44 & 5.98 & 5.60 & 5.26 & 6.05 \\
\hline EC-608415 × EC-520078 & 8.03 & 7.89 & 7.43 & 7.36 & 7.32 & 7.61 \\
\hline EC-608415 × EC-620557 & 2.51 & 2.15 & 1.87 & 1.63 & 1.57 & 3.20 \\
\hline EC-619982 × Arka Saurabh & 3.52 & 3.43 & 2.26 & 1.59 & 1.54 & 2.19 \\
\hline EC-619982 × Arka Abha & 8.78 & 8.12 & 7.50 & 6.71 & 6.63 & 6.50 \\
\hline EC-619982 × Punjab Chhuhara & 7.98 & 6.43 & 4.10 & 3.00 & 2.51 & 4.80 \\
\hline EC-619982 × Pusa Ruby & 5.60 & 4.34 & 3.99 & 3.67 & 2.36 & 3.99 \\
\hline EC-619982 × EC-520078 & 4.96 & 3.73 & 3.32 & 2.69 & 2.40 & 3.42 \\
\hline EC-619982 × EC-620557 & 5.24 & 4.28 & 3.62 & 3.57 & 2.21 & 3.78 \\
\hline US-440 & 9.87 & 8.24 & 8.20 & 8.13 & 5.26 & 7.94 \\
\hline Arka Rakshak & 9.98 & 9.41 & 8.34 & 8.02 & 7.92 & 8.73 \\
\hline NS-516 & 5.99 & 5.43 & 4.88 & 4.68 & 4.29 & 5.05 \\
\hline \multirow[t]{2}{*}{ Mean } & 6.11 & 5.18 & 4.64 & 4.16 & 3.67 & \\
\hline & \multicolumn{3}{|c|}{ S.Em \pm} & \multicolumn{3}{|c|}{ CD (0.01) } \\
\hline Treatment (A) & \multicolumn{3}{|c|}{0.081} & \multicolumn{3}{|c|}{0.226} \\
\hline Genotype (B) & \multicolumn{3}{|c|}{0.032} & \multicolumn{3}{|c|}{0.088} \\
\hline $\mathbf{A} \times \mathbf{B}$ & \multicolumn{3}{|c|}{0.182} & \multicolumn{3}{|c|}{0.506} \\
\hline
\end{tabular}


Table.6 Effect of different concentration of $\mathrm{NaCl}$ (control, 50mM, 75mM, $100 \mathrm{M}$ and 150mM on root dry weight $(\mathrm{mg})$ of tomato genotypes

\begin{tabular}{|c|c|c|c|c|c|c|}
\hline \multirow{3}{*}{ Crosses/Hybrids } & \multicolumn{6}{|c|}{ Root dry weight (mg) } \\
\hline & \multicolumn{6}{|c|}{ Treatments } \\
\hline & Control & $\begin{array}{c}\mathbf{5 0 m} \\
\mathrm{M} \\
\end{array}$ & $75 \mathrm{mM}$ & $100 \mathrm{mM}$ & $150 \mathrm{mM}$ & Mean \\
\hline EC-620407 × Arka Saurabh & 6.95 & 6.23 & 6.01 & 5.58 & 5.52 & 4.56 \\
\hline EC-620407 × Arka Abha & 3.32 & 2.88 & 2.79 & 2.83 & 2.58 & 4.38 \\
\hline EC-620407 × Punjab Chhuhara & 10.27 & 9.36 & 8.78 & 4.06 & 3.87 & 6.43 \\
\hline EC-620407 × Pusa Ruby & 3.20 & 2.34 & 1.90 & 1.85 & 1.80 & 3.05 \\
\hline EC-620407 × EC-520078 & 7.69 & 7.06 & 5.90 & 5.46 & 5.19 & 6.26 \\
\hline EC-620407 × EC-620557 & 9.55 & 8.33 & 5.42 & 4.12 & 0.93 & 5.08 \\
\hline EC-620428 × Arka Saurabh & 2.77 & 2.67 & 1.43 & 1.17 & 0.90 & 2.38 \\
\hline EC-620428 × Arka Abha & 3.73 & 3.00 & 2.99 & 2.32 & 1.98 & 2.80 \\
\hline EC-620428 × Punjab Chhuhara & 7.55 & 5.82 & 5.29 & 4.10 & 2.75 & 5.10 \\
\hline EC-620428 × Pusa Ruby & 4.14 & 3.77 & 3.52 & 3.11 & 1.22 & 3.70 \\
\hline EC-620428 × EC-520078 & 5.37 & 5.17 & 4.60 & 3.95 & 3.75 & 4.02 \\
\hline EC-620428 × EC-620557 & 5.47 & 4.94 & 4.44 & 3.93 & 3.80 & 3.97 \\
\hline EC-620360 × Arka Saurabh & 2.96 & 1.62 & 1.47 & 1.95 & 1.08 & 2.36 \\
\hline EC-620360 × Arka Abha & 5.21 & 4.46 & 3.70 & 2.10 & 1.92 & 3.48 \\
\hline EC-620360 × Punjab Chhuhara & 2.97 & 2.82 & 2.37 & 2.19 & 1.95 & 2.46 \\
\hline EC-620360 × Pusa Ruby & 2.92 & 2.19 & 1.76 & 1.75 & 1.88 & 2.10 \\
\hline EC-620360 × EC-520078 & 4.37 & 3.19 & 2.84 & 2.37 & 1.81 & 2.92 \\
\hline EC-620360 × EC-620557 & 4.21 & 3.43 & 3.01 & 2.95 & 2.04 & 3.13 \\
\hline EC-608415 × Arka Saurabh & 3.44 & 3.25 & 2.59 & 2.41 & 2.23 & 2.78 \\
\hline EC-608415 × Arka Abha & 5.30 & 4.00 & 3.04 & 2.49 & 1.92 & 2.98 \\
\hline EC-608415 × Punjab Chhuhara & 2.94 & 2.20 & 2.16 & 1.60 & 0.93 & 2.34 \\
\hline EC-608415 × Pusa Ruby & 5.88 & 4.17 & 3.03 & 2.81 & 2.33 & 3.64 \\
\hline EC-608415 × EC-520078 & 5.19 & 5.33 & 3.88 & 3.05 & 2.97 & 4.08 \\
\hline EC-608415 × EC-620557 & 5.67 & 4.89 & 4.03 & 3.14 & 2.77 & 4.10 \\
\hline EC-619982 × Arka Saurabh & 4.34 & 3.31 & 3.25 & 3.09 & 2.91 & 3.38 \\
\hline EC-619982 × Arka Abha & 5.06 & 3.96 & 3.72 & 3.53 & 2.73 & 3.80 \\
\hline EC-619982 × Punjab Chhuhara & 5.15 & 4.80 & 4.41 & 4.26 & 4.13 & 4.55 \\
\hline EC-619982 × Pusa Ruby & 8.85 & 6.02 & 5.92 & 4.30 & 3.87 & 5.23 \\
\hline EC-619982 × EC-520078 & 7.54 & 6.02 & 4.37 & 3.81 & 3.17 & 5.55 \\
\hline EC-619982 × EC-620557 & 7.53 & 5.37 & 5.36 & 3.80 & 3.23 & 5.06 \\
\hline US-440 & 10.95 & 10.76 & 9.90 & 9.44 & 8.56 & 9.92 \\
\hline Arka Rakshak & 11.37 & 9.92 & 9.56 & 8.80 & 8.25 & 9.58 \\
\hline NS-516 & 11.61 & 10.55 & 10.06 & 9.53 & 8.88 & 10.13 \\
\hline \multirow[t]{2}{*}{ Mean } & 5.86 & 4.96 & 4.35 & 3.69 & 3.15 & 4.40 \\
\hline & \multicolumn{3}{|c|}{ S.Em \pm} & \multicolumn{3}{|c|}{ CD (0.01) } \\
\hline Treatment (A) & \multicolumn{3}{|c|}{0.084} & \multicolumn{3}{|c|}{0.233} \\
\hline Genotype (B) & \multicolumn{3}{|c|}{0.033} & \multicolumn{3}{|c|}{0.091} \\
\hline $\mathbf{A} \times \mathbf{B}$ & \multicolumn{3}{|c|}{0.187} & \multicolumn{3}{|c|}{0.521} \\
\hline
\end{tabular}


Table.7 Effect of different concentration of $\mathrm{NaCl}$ (control, 50mM, 75mM, $100 \mathrm{M}$ and 150mM on shoot dry weight $(\mathrm{mg})$ of tomato genotypes

\begin{tabular}{|c|c|c|c|c|c|c|}
\hline \multirow{3}{*}{ Crosses/Hybrids } & \multicolumn{6}{|c|}{ Shoot dry weight (mg) } \\
\hline & \multicolumn{6}{|c|}{ Treatments } \\
\hline & Control & $50 \mathrm{mM}$ & $\begin{array}{c}75 \mathrm{~m} \\
\mathrm{M}\end{array}$ & $100 \mathrm{mM}$ & $150 \mathrm{mM}$ & Mean \\
\hline EC-620407 × Arka Saurabh & 46.36 & 42.93 & 32.56 & 28.15 & 25.99 & 35.20 \\
\hline EC-620407 × Arka Abha & 32.61 & 27.89 & 23.96 & 18.26 & 15.01 & 23.54 \\
\hline EC-620407 × Punjab Chhuhara & 66.99 & 65.23 & 60.93 & 50.60 & 18.87 & 45.67 \\
\hline EC-620407 × Pusa Ruby & 24.12 & 22.25 & 19.50 & 14.33 & 11.72 & 28.44 \\
\hline EC-620407 × EC-520078 & 81.44 & 76.12 & 72.83 & 67.69 & 24.70 & 64.55 \\
\hline EC-620407 × EC-620557 & 72.04 & 71.37 & 59.24 & 44.59 & 35.81 & 66.61 \\
\hline EC-620428 × Arka Saurabh & 27.07 & 22.76 & 21.19 & 19.32 & 13.69 & 30.80 \\
\hline EC-620428 × Arka Abha & 31.01 & 30.53 & 28.68 & 26.03 & 17.26 & 34.70 \\
\hline EC-620428 × Punjab Chhuhara & 56.11 & 54.66 & 43.74 & 32.30 & 15.73 & 46.51 \\
\hline EC-620428 × Pusa Ruby & 42.75 & 35.53 & 28.99 & 26.86 & 14.61 & 32.95 \\
\hline EC-620428 × EC-520078 & 54.03 & 53.64 & 46.96 & 42.61 & 20.80 & 51.01 \\
\hline EC-620428 × EC-620557 & 73.67 & 71.59 & 63.92 & 37.11 & 25.87 & 61.43 \\
\hline EC-620360 × Arka Saurabh & 45.69 & 34.64 & 31.45 & 26.24 & 20.11 & 27.43 \\
\hline EC-620360 × Arka Abha & 82.30 & 78.39 & 71.96 & 65.03 & 29.95 & 72.92 \\
\hline EC-620360 × Punjab Chhuhara & 30.67 & 28.64 & 24.23 & 22.53 & 17.73 & 24.76 \\
\hline EC-620360 × Pusa Ruby & 40.86 & 39.14 & 35.36 & 32.20 & 15.85 & 42.68 \\
\hline EC-620360 × EC-520078 & 51.74 & 43.31 & 41.96 & 37.66 & 19.04 & 38.74 \\
\hline EC-620360 × EC-620557 & 58.63 & 47.78 & 45.88 & 41.40 & 30.07 & 44.75 \\
\hline EC-608415 × Arka Saurabh & 88.07 & 82.49 & 66.08 & 50.68 & 30.92 & 69.65 \\
\hline EC-608415 × Arka Abha & 38.71 & 36.41 & 33.55 & 31.26 & 26.54 & 33.29 \\
\hline EC-608415 × Punjab Chhuhara & 79.38 & 50.40 & 49.07 & 43.04 & 15.41 & 51.46 \\
\hline EC-608415 × Pusa Ruby & 31.12 & 30.48 & 28.00 & 24.45 & 19.78 & 27.96 \\
\hline EC-608415 × EC-520078 & 43.41 & 41.10 & 40.51 & 32.39 & 20.36 & 38.76 \\
\hline EC-608415 × EC-620557 & 49.63 & 43.26 & 41.41 & 34.52 & 24.92 & 38.75 \\
\hline EC-619982 × Arka Saurabh & 80.31 & 79.74 & 70.88 & 39.03 & 22.38 & 66.47 \\
\hline EC-619982 × Arka Abha & 70.10 & 68.36 & 64.03 & 45.79 & 32.92 & 60.24 \\
\hline EC-619982 × Punjab Chhuhara & 29.75 & 29.27 & 25.78 & 22.78 & 13.08 & 35.40 \\
\hline EC-619982 × Pusa Ruby & 61.39 & 35.62 & 31.49 & 24.24 & 16.72 & 40.09 \\
\hline EC-619982 × EC-520078 & 49.80 & 44.16 & 39.65 & 22.38 & 12.02 & 40.40 \\
\hline EC-619982 × EC-620557 & 50.48 & 50.13 & 40.21 & 30.20 & 19.79 & 46.16 \\
\hline US-440 & 85.86 & 83.10 & 64.16 & 53.15 & 29.72 & 83.20 \\
\hline Arka Rakshak & 86.48 & 84.18 & 64.30 & 44.09 & 20.76 & 83.96 \\
\hline NS-516 & 82.71 & 77.80 & 75.05 & 34.03 & 22.51 & 84.42 \\
\hline \multirow[t]{2}{*}{ Mean } & 55.92 & 51.00 & 45.08 & 35.30 & 21.23 & \\
\hline & \multicolumn{3}{|c|}{ S.Em \pm} & \multicolumn{3}{|c|}{ CD (0.01) } \\
\hline Treatment (A) & \multicolumn{3}{|c|}{0.681} & \multicolumn{3}{|c|}{1.895} \\
\hline Genotype (B) & \multicolumn{3}{|c|}{0.265} & \multicolumn{3}{|c|}{0.738} \\
\hline$A \times B$ & \multicolumn{3}{|c|}{1.523} & \multicolumn{3}{|c|}{0.438} \\
\hline
\end{tabular}


After radicle emergence, hypocotyl emerges and lifts the growing tip above the ground. Under drought stress condition, the root develops faster than the hypocotyls to acclimatize the drought stress. Therefore, the growth of radicle and hypocotyls should reflect the adaptability of plant to drought stress (Zhu et al., 2006).

Significant differences were observed for root length between the genotypes and different $\mathrm{NaCl}$ concentrations (Table 4). Irrespective of the salt concentrations, EC-620428 $\times$ Pusa Ruby recorded significantly maximum root length $(7.56 \mathrm{~cm})$ compared to all others. Root length was lowest $(1.87 \mathrm{~cm})$ in the genotype EC-619982 $\times$ EC-520078. At 50mM concentration of $\mathrm{NaCl}$, maximum and minimum root length was recorded in EC$620428 \times$ Pusa Ruby $(8.16 \mathrm{~cm})$ and EC$619982 \times$ EC-520078 $(2.09 \mathrm{~cm})$ respectively. While at $75 \mathrm{mM}$ concentration, genotypes NS$516(7.58 \mathrm{~cm})$ and EC-619982 × EC-520078 $(1.99 \mathrm{~cm})$ exhibited maximum and minimum root lengths. With $100 \mathrm{mM}$ concentration of $\mathrm{NaCl}$, genotypes EC-620428 $\times$ Pusa Ruby $(7.05 \mathrm{~cm})$ and EC-619982 $\times$ EC-520078 (1.39 $\mathrm{cm})$ responded as maximum and minimum root lengths. In case of $150 \mathrm{mM}$ concentration, genotypes EC-620428 × Pusa Ruby $(6.82 \mathrm{~cm})$ and EC-619982 $\times$ EC-520078 $(1.26 \mathrm{~cm})$ responded as maximum and minimum root length respectively. Similar results were observed by Souguir et al., (2013) Aamir et al., (2012).

\section{Shoot Length (cm)}

The results revealed significant difference in shoot length between the genotypes and different $\mathrm{NaCl}$ concentrations (Table 5). Irrespective of salt concentrations, EC$620360 \times$ EC-620557 recorded significantly maximum shoot length $(9.49 \mathrm{~cm})$ while lowest $(2.27 \mathrm{~cm})$ was in EC-620428 $\times$ Pusa Ruby among all genotypes. At $50 \mathrm{mM}$ concentration of $\mathrm{NaCl}$, maximum and minimum shoot length was recorded in EC$620360 \times$ EC-620557 $(10.09 \mathrm{~cm})$ and EC$620360 \times$ Arka Abha $(1.60 \mathrm{~cm})$ genotypes respectively. While at $75 \mathrm{mM}$ concentration of $\mathrm{NaCl}$, genotype EC-620360 × EC-620557 $(8.93 \mathrm{~cm})$ and EC-608415 x Arka Abha (1.40 $\mathrm{cm})$ exhibited maximum and minimum shoot lengths. In case of $100 \mathrm{mM}$ concentration, genotypes EC-620360 × EC-620557 (8.91 $\mathrm{cm})$ and EC-620360 $\times$ Arka Abha $(1.32 \mathrm{~cm})$ responded with maximum and minimum shoot length respectively. With $150 \mathrm{mM}$ concentration of $\mathrm{NaCl}$, genotypes EC-620360 $\times$ EC-620557 $(8.91 \mathrm{~cm})$ and EC-608415 $\times$ Arka Abha $(0.41 \mathrm{~cm})$ recorded with maximum and minimum shoot length respectively.

Genotypes with indeterminate growth habit showed more reduction in shoot length as compared to determinate type. It indicates that determinate tomato can be well suited to drought areas than indeterminate growth habit. Present experimental results were similar to earlier studies of Aamir et al., (2012).

\section{Root dry weight (mg)}

Highest root weight was exhibited by NS-516 (10.13) (Table 6). Regardless of the salt concentrations, root dry weight was lowest (2.10) in EC-620360 × Pusa Ruby. In 50mM concentration of $\mathrm{NaCl}$, maximum and minimum root dry weight was recorded in NS-516 (10.55 mg) and EC-620360 × Pusa Ruby $(2.19 \mathrm{mg})$ respectively. While at $75 \mathrm{mM}$ concentration, genotypes NS-516 (10.06 mg) and EC-620428 × Arka Saurabh (1.43 mg) exhibited maximum and minimum root dry weight respectively. In case of $100 \mathrm{mM}$ concentration of $\mathrm{NaCl}$, genotypes NS-516 (9.53 mg) and EC-620428 × Arka Saurabh (1.17 $\mathrm{mg}$ ) had responded as maximum and minimum root dry weight respectively. With 
$150 \mathrm{mM}$ concentration of $\mathrm{NaCl}$, genotypes NS-516 $(8.88 \mathrm{mg})$ and EC-620428 $\times$ Arka Saurabh $(0.90 \mathrm{~cm})$ had responded as maximum and minimum root dry weight respectively

Growth parameters like dry weight is known to have a profound effect on water limited conditions. In the present study a reduction in root dry weight was recorded in stressed conditions in all the genotypes. Present investigation is in confirmation with Ahmet et al., (2009) and Soughir et al., (2013). Root dry weight depends on the germination percent and root length, low water uptake and restricted metabolic activities were given to decrease in the root dry weight. Most of the tolerant genotypes accumulated more dry matter under control that too increased under stress however moderately tolerant or susceptible genotypes recorded comparatively less increase or even decrease in dry weight of roots.

\section{Shoot dry weight (mg)}

Irrespective of the $\mathrm{NaCl}$ concentrations, NS516 recorded significantly maximum shoot dry weight $(84.42 \mathrm{mg})$ among all genotypes (Table 7) with lowest $(23.54 \mathrm{mg})$ in EC$620407 \times$ Arka Abha. In 50mM concentration, maximum and minimum shoot dry weight was recorded in Arka Rakshak (84.18 mg) and EC-620407 x Pusa Ruby $(22.25 \mathrm{mg})$ respectively. While at $75 \mathrm{mM}$ concentration of $\mathrm{NaCl}$, genotype NS-516 $(75.05 \mathrm{mg})$ and EC-620407 $\times$ Pusa Ruby $(19.50 \mathrm{mg})$ exhibited maximum and minimum shoot dry weight. With $100 \mathrm{mM}$ concentration EC-620407 $\times$ EC-520078 (67.69 mg) and EC$620407 \times$ Pusa Ruby (14.33 mg) had responded with maximum and minimum shoot dry weight respectively. In case of $150 \mathrm{mM}$ concentration of $\mathrm{NaCl}$, genotypes EC-620407 x EC-620557 (35.81 mg) and EC$620407 \times$ Pusa Ruby (11.72 mg) had responded with maximum and minimum shoot dry weight.

High levels of salinity reduced shoot dry weight. This is probably due to the reaction of salinity, which reduces the contribution of phytohormones in the biosynthesis (Cuartero et al., 2006). These results are in accordance with Ahmet et al., (2009) and Soughir et al., (2013).

Salinity is a major constraint to economic use of land for agriculture especially in the coastal regions. Among the commercial checks NS516 while among crosses EC-619982 $\times$ EC520078, EC-619982 × Pusa Ruby, EC-620428 $\times$ Punjab Chhuhara, EC-620360 $\times$ EC620557, EC-620407 $\times$ Punjab Chhuhara and EC-608415 $\times$ Arka Saurabh responded positively against different $\mathrm{NaCl}$ concentrations. The genotypes can be further tested under pot culture studies and if found promising field level experiments may be carried out for confirming their potential.

\section{References}

Aamir Nawaz, Muhammad Amjad, Muhammad Muzammil Jahangir, Samiya Mahmood Khan, Huawei Cui and Jin Hu. 2012. Induction of salt tolerance in tomato (Lycopersicon esculentum Mill.) seeds through sand priming. Australian Journal of Crop Science. 6(7):1199-1203.

Ahmet Turhan, Vedat eniz and Hayrettin Kuscu. 2009. Genotypic variation in the response of tomato to salinity. African Journal of Biotechnology. 8:6. 10621068.

Alejandra Nieto-Garibay, Bernardo MurilloAmador, Enrique Troyo-Dieguez, Jose L. Garcia-Hernandez and Francisco H. Ruiz-Espinoza. 2010. Water stress in two capsicum species with different domestication grade. Tropical and 
Subtropical Agroecosystems. 12: 353 360.

Allen, R.G., Pereira, L.S. Raes, D. and Smith. M. 1998. Crop evapotranspiration: Guidelines for computing crop water requirements. Irrigation \& Drainage. Paper 56. UN-FAO, Rome, Italy.

Babu, L., Muthukrishnan, C. R. and Irulappan I. 1985. Studies on drought tolerance in tomato. South Indian Horticulture. 33: 377-380.

Caro, M., Cruz, V., Cuartero, J., Estañ, M.T. and Bolarin, M.C. 1991. Salinity tolerance of normal-fruited and cherry tomato cultivars. Plant and Soil. 136: 249- 255.

Cuartero J., Bolarin M.C., Asins M.J., Moreno V., 2006. Increasing salt tolerance in tomato. Journal of Experimental Botany. 57: 1045-1058.

El-Saifi, S. K., H. M. I. Ahmed, Sawsan M. Hasan, M. M. Morsi and Rowaa S. ElShatoury. 2010. J. of Plant Production, Vol. 1 (2): 159 - 170.

Flowers, T.J. (2004) Improving crop salt tolerance. J. Exp. Bot. 55: 307-319.

Foolad, M. R., J. R. Hyman and G. Y. Lin. 1999. Relationships between cold-and salt-tolerance during seed germination in tomato: analysis of response and correlated response to selection. Plant Breeding: 118: 49-52.

Foolad, M.R. 2007 Genome mapping and molecular breeding of tomato. International Journal of Plant Genomics. 64358.

Hasanuzzaman, M., K. Nahar, Md.M. Alam, P.C. Bhowmik, Md.A. Hossain, M.M. Rahman, M.N.V. Prasad, M. Ozturk and M. Fujita. 2014. Potential use of halophytes to remediate saline soils. Biomed Research Institute. 589341.

Karlberg, L., Ben-Gal, A., Jansson, P-E. and Shani, U. 2006. Modeling transpiration and growth in salinity-stressed tomato under different climatic conditions.
Ecological Modeling, 190: 15-40.

Kim, Y. J. Shanmugasundaram, S. Yun, S. J. Park, H. K. and Park M.S. 2001. A simple method of seedling screening for drought tolerance in soybean. Korean Journal of Crop Science. 46: 284-288.

Narayan, D. 1991. Root growth and productivity of wheat cultivars under different soil moisture conditions. International Journal of Ecology and Environmental Science. 17: 19-26.

Panchalingam, S. 1983. Studies on drought tolerance in brinjal (Solanum melongena L.) M.Sc (Hort.) Thesis, TNAU, Coimbatore, India.

Parida A.K. and Das A.B. 2005. Salt tolerance and salinity effects on plant: a review. Ecotoxical Environ Safety. 60:324-349.

Peralta, E., Knapp, S. and Spooner, O.M. 2005. New species of wild tomato (Solanum section Lycoperisicon: Solanaceae) flom; Iorthem Pem. Systematic Bot., 30: 424-434.

Ravi Ranjan Kumar, Karjol, K. and Naik G. R. 2011. Variation of sensitivity to drought stress in Pigeon pea (Cajanus cajan (L) cultivars during seed germination and early seedling growth. World Journal of Science and Technology. 1(1): 11-18.

Sivritepe, N., H.O. Sivritepe and A. Erifl. 2003. The effects of $\mathrm{NaCl}$ priming on salt tolerance in melon seedlings grown under saline conditions. Scientia Hort. 97: 229-237.

Sonneveld, C. and Van der Burg, A.M.M. 1991. Sodium chloride salinity in fruit vegetable crops in soilless culture. Netherlands J. Agri. Sci., 39: 115-122.

Soughir M, Elouaer M.A., Hannachi C. 2013. The Effect of $\mathrm{NaCl}$ Priming on emergence, growth and yield of fenugreek under saline conditions. Cercetări Agronomice în Moldova. Vol. XLVI, No. 2 (154):73-83. 
Steel, R.G.D., J.H. Torrie and Dickey D.A. 1997. Principles and Procedures of Statistics: A Biometrical Approach. 3rd Ed. McGraw Hill Book Co. Inc. New York, USA.

Uniyal, R. C. and Nautiyal A. R. 1998. Seed germination and seedling extension growth in Ougeinia dalbergioides benth. under water and salinity stress.
New Forests. 16: 265-272.

Zhu, J. Kang, H. Tan, H. and Xu M. 2006. Effects of drought stresses induced by polyethylene glycol on germination of Pinus sylvestris var. mongolica seeds from natural and plantation forests on sandy land. Journal of Forest Research. 11: 319-328.

\section{How to cite this article:}

Arun Kumar P., N. N. Reddy and Jyothi Lakshmi N. 2017. Screening Tomato Genotypes for Salt Tolerance. Int.J.Curr.Microbiol.App.Sci. 6(11): 1037-1049. doi: https://doi.org/10.20546/ijcmas.2017.611.xx 